\title{
5-Year Survival Rates and Prognostic Factors in Patients with Synchronus and Metachronus Breast Cancer from 2010 to 2015
}

\author{
Arash Sarveazad ${ }^{1}$, Asrin Babahajian², Jebreil Shamseddin ${ }^{3}$, Mansour \\ Bahardoust $^{1 *}$
}

\begin{abstract}
Introduction: Currently breast $(\mathrm{BC})$ cancer is a serious medical problem in all countries of the world. Survival depends on many factors. The present study focused on 5-year survival and its related factors in patients with BC in Iran. Material and methods. The present analytical retrospective study was performed (from March 2010 until March 2015) on patients with BC followed for at least 6 months. The main variables assessed were tumor size, grade of lymph node involvement, metastasis, stage, history, human epidermal growth factor receptor expression, and tumor origin. Analysis of survival was accomplished using the Kaplan- Meier method. Results: Some $351(80.2 \%)$ of the total of 438 individuals had unilateral and $87(19.8 \%)$ had bilateral cancer, $28(35.6 \%)$ of the latter being synchronous and $56(64.4 \%)$ metachronous. Mean duration of follow-up was $47.44 \pm 28.19$ months, during which 61 (17.3\%) patients with unilateral and 18 with bilateral cancer eventually died. The 5-year survival rate in patients with unilateral BC was significantly higher than those with bilateral BC (Log-rank Test chi2=3.11, $\mathrm{p}=0.032$ ). In addition, with metachronous cases, the survival rate was $64.2 \%$ in comparison with $51.6 \%$ for synchronous BCs. Survival rate was significantly ( $p$ value $=0.038)$ higher with metachronous than with synchronous cancers (Log-rank Test chi2 $=3.54, p=0.038)$. The highest survival rate was reported for BCs originating from lobule tissue and the lowest rate examples of interstitial tissue origin (Log-rank Test chi2 $=11.54, \mathrm{p}=0.0001$ ). Patients with earl stage lesions $(\mathrm{M} 1)$ survived longer than with other stages (Log-rank Test chi2 $=9.55, \mathrm{p}=0.001$ ). Conclusion: In this study, most women with BC had a positive family history and were married. The 5-year survival rate was lower with advanced stages of cancer. According to our findings, survival rates might improve if patients undergo screening and diagnosis is made at an early stage of the disease.
\end{abstract}

Keywords: Breast cancer- survival- metastasis- clinical stage- Iran

Asian Pac J Cancer Prev, 19 (12), 3489-3493

\section{Introduction}

Currently, cancer is a serious and prevalent problem that all of the countries of the world are faced with. In 2008, 12.7 million cancer cases and 7.6 million cases of death due to cancer were reported from around the world (Jemal et al., 2011). The epidemiologic pattern of cancer varies in different countries and $60 \%$ of all cancers are detected in developing countries such as African and Asian countries (Vakili et al., 2014). Prevalence of various types of cancer is different between men and women (Dorak and Karpuzoglu, 2012). Unlike the past decade in which cervical cancer was the most common cancer among women, currently, the most prevalent one is breast cancer (BC); $23 \%$ of all new cases of cancer and $14 \%$ of deaths due to cancer belong to BC (Jemal et al., 2011). $\mathrm{BC}$ in women affects their quality of life intensely and inflicts a great deal of mental and financial burden on the patient, the patient's relatives and the society. Based on the statistics announced by National Institute of Health in 2005, the expenses of BC were about 209.9 billion Dollars (Brown et al., 2001). In cases that BC leads to death of the patients, duration of survival depends on many factors such as tumor diameter, lymph node metastasis (LNM) and its stage (Dong et al., 2014). Predicting the survival of patients after being diagnosed with $\mathrm{BC}$ and evaluating the correlation of various factors with its duration has been the subject of many studies in the field of epidemiology. Numerous pathologic indices such as stage of tumor, involvement of lymph nodes, and tumor receptor have been identified and confirmed as markers of cancer prognosis in BC (Balabram et al., 2013; Nishimura et al., 2013). However, since the epidemiologic pattern of cancer varies in different countries (Vakili et al., 2014) and the correlation between these prognostic markers and rate of survival after BC diagnosis has not been evaluated in Iran, the present study has assessed the 5-year survival rate and its related factors in patients with $\mathrm{BC}$ presenting

${ }^{1}$ Colorectal Research Center, Iran University of Medical Sciences, Tehran, ${ }^{2}$ Liver and Digestive Research Center, Kurdistan University of Medical Sciences, Sanandaj, ${ }^{3}$ Infectious and Tropical Diseases Research Center, Hormozgan Health Institute, Hormozgan University of Medical science, Bandar Abbas, Iran.*For Correspondence: mansourbahari93@gmail.com 
to Rasoul e Akram Hospital, Tehran, Iran, from March 2010 until March 2015.

\section{Materials and Methods}

Design

The present analytical retrospective study was performed in Iran University of Medical sciences, Tehran, Iran.

\section{Patients}

All the patients with $\mathrm{BC}$ who visited Rasoul e Akram Hospital, affiliated with Iran University of Medical Sciences, over a 5-year period from March 2010 until March 2015 for undergoing surgery to remove the tumor were studied. To diagnose metastasis in patients, either of biochemical methods, ultrasound, or increase in blood markers was used. The final diagnosis of metastasis was done by an oncologist.

\section{Data gathering}

Demographic and clinical data of the patients were gathered from the archiving unit of the hospital and pathologic data were obtained from the pathology laboratory of the hospital. Data regarding the follow-up of patients has been recorded in their profile.

\section{Eligibility criteria and follow-up}

For inclusion in the study, the patients were followed for at least 6 months. The follow-up of patients was continued until the end of the study period or until the patient died.

In patients with bilateral $\mathrm{BC}$, if the time interval between diagnosis of cancer in the initially healthy breast was 6 months or less after the affected breast, the cancer was defined as a synchronous bilateral $\mathrm{BC}$ and diagnosis more than 6 months after the other was defined as metachronous bilateral $\mathrm{BC}$.

The main variables in 5-year survival assay consist of tumor size, grade of lymph node involvement $(\mathrm{N} 1, \mathrm{~N} 2$ or N3), presence of metastasis, stage of cancer, age of being affected with cancer, history of $\mathrm{BC}$ in first degree relatives, human receptor of epidermal growth factor (HER2) and tumor origin tissue consist of Invasive ductal carcinoma (IDC), Ductal carcinoma in situ (DCIS) and Invasive lobular carcinoma (ILC), and covariates consist of age of the patient at the time of surgery, family history and marital status. Finally, the survival duration of the patients was recorded.

The final status of the patient regarding survival or metastasis occurrence was gathered via phone calls. Patients who had an undetermined situation in followup were excluded from the study. The main variables and covariates in survival of patients with unilateral and bilateral cancers were compared.

\section{Statistical Analysis}

Survival rate in the studied population has been calculated by dividing the number of individuals who survived after 5 years of follow-up to the total number of the patients. To draw the survival rate curve, the KaplanMeier method was applied. To compare variables between the 2 groups, after normality test (Kolmogorov-Smirnov) parametric $\mathrm{T}$ test or non-parametric Mann-Whitney test were used for quantitative variables (shown as mean $\pm \mathrm{SD}$ ) and chi square test was used for comparing qualitative variables. Kaplan-Meier test was applied to draw the survival curve, and log-rank test was used for determining the difference between survival curves by eliminating patients whose statuses were undetermined. P-values less than 0.05 were considered statistically significant. Statistical analyses were performed by SPPS software version 22 .

\section{Results}

By reviewing medical profiles of the patients from March 2010 until March 2015, a total of 438 BC patients admitted to Rasoul e Akram Hospital whose profiles were available for follow-up, were included in the study. 351 $(80.2 \%)$ of the total 438 individuals had unilateral cancer and $87(19.8 \%)$ were affected with bilateral cancer. Mean duration of follow-up was $47.44 \pm 28.19$ months (from 6 months to 92 months). The average age of the patients at the time of diagnosis was reported as $46.88 \pm 19.2$ years among those with unilateral cancer and $47.20 \pm 20.1$ years in those with bilateral cancer. 104 (29.6\%) of the patients with unilateral cancer were positive and $35.6 \%$ of bilateral
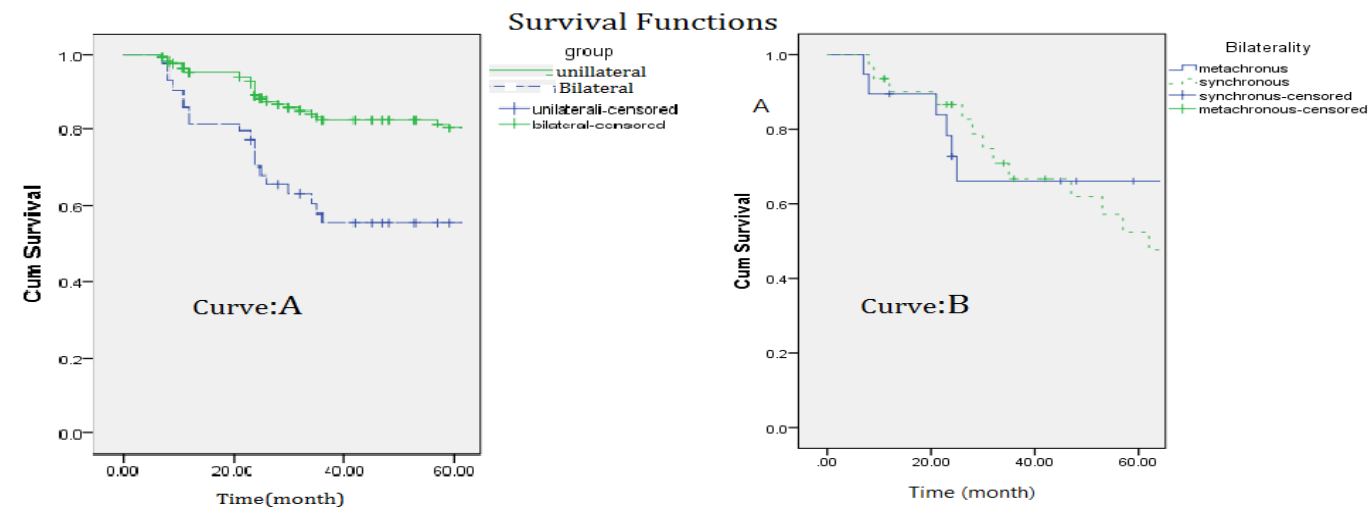

Figure 1. A) The curve comparing survival in the 2 groups of patients with unilateral breast cancer and patients with bilateral breast cancer B) The curve comparing survival in the 2 groups of patients with bilateral synchronous and bilateral metachronous breast cancer 
DOI:10.31557/APJCP.2018.19.12.3489

5-Year Survival Rates and Survival Predictors in Patients

Table 1. Demographic Variables of the Patients in the 2 Groups

\begin{tabular}{lccc}
\hline Variable & $\begin{array}{c}\text { Unilateral } \\
\text { cancer }\end{array}$ & $\begin{array}{c}\text { Bilateral } \\
\text { cancer }\end{array}$ & $\begin{array}{c}P \text { - } \\
\text { value }\end{array}$ \\
\hline Number n(\%) & $351(80.2 \%)$ & $87(19.8)$ & - \\
Age (mean \pm sd) & $(46.88 \pm 19.2)$ & $(47.20 \pm 20.1)$ & 0.6 \\
Family history n(postive)\% & $104(29.6 \%)$ & $31(35.6 \%)$ & 0.77 \\
Married n\%(yes) & $289(82.3 \%)$ & $76(87.3 \%)$ & 0.098 \\
\hline
\end{tabular}

cases were positive regarding family history of cancer (Table1)

Most of the tumors were diagnosed in M2 stage (spread of cancer from one part of the body to another) ( $p$ value $=0.001$ ). Diagnosed tumor size was between 2 and $5 \mathrm{~cm}$ ( $\mathrm{p}$ value $=0.023$ ). Regarding the rate of lymph node involvement, N2 was the significantly more prevalent status reported among the patients ( $p$ value $=0.027$ ).

IDC was the most prevalent histology of tumor among patients with unilateral and bilateral cancers ( $\mathrm{p}$ value $=$ 0.001). The status of HER2 receptor was reported as unknown in most patients and no significant correlation was detected between the survival rate of the patients and HER2 receptor $(\mathrm{p}$ value $=0.7)$.

In patients with metachronous $\mathrm{BC}$, the average time interval between the first breast being affected and diagnosis of cancer in the initially healthy breast was reported as 61.8 months. Clinical and pathologic states of the patients have been presented in Table 2 .

To perform and report the analysis of survival, Kaplan- Meier method was used. 61 (17.3\%) patients with unilateral cancer and 35(40.2\%) patients with bilateral BC (21 in the metachronous group and 14 in the synchronous group) had died. 5-year survival rate in patients with unilateral breast cancer was significantly more than those with bilateral breast cancer (Figure 1 curve (A), Log-rank Test $\mathrm{chi} 2=3.11, \mathrm{p}=0.032$ ).

In comparison of survival rate in patients with bilateral $\mathrm{BC}$, the survival rate of patients with metachronous cancer was reported to be significantly higher than those with synchronous cancer $(\mathrm{p}$ value $=0.001)$

5 -year survival rate in patients with metachronous bilateral breast cancer was $64.2 \%$ while it was $51.6 \%$ for
Table 2. Clinical and Pathologic States of the Patients in the 3 Groups

\begin{tabular}{|c|c|c|c|c|}
\hline \multirow[t]{2}{*}{ Variable } & \multirow{2}{*}{$\begin{array}{c}\text { Unilateral } \\
\text { cancer (n\%) }\end{array}$} & \multicolumn{2}{|c|}{ Bilateral cancer } & \multirow{2}{*}{$\begin{array}{c}P \\
\text { value }\end{array}$} \\
\hline & & $\begin{array}{c}\text { Synchronous } \\
(\mathrm{n} \%)\end{array}$ & $\begin{array}{c}\text { Metachronous } \\
(\mathrm{n} \%)\end{array}$ & \\
\hline $\mathrm{N}$ & & & & 0.027 \\
\hline 1 & $53(15 \%)$ & $7(22.5 \%)$ & $16(28 \%)$ & \\
\hline 2 & $254(73 \%)$ & $21(68 \%)$ & $33(59 \%)$ & \\
\hline 3 & $44(12 \%)$ & $3(9.5 \%)$ & $8(13 \%)$ & \\
\hline M & & & & 0.001 \\
\hline 1 & $92(26 \%)$ & $8(25 \%)$ & $10(18 \%)$ & \\
\hline 2 & $206(59 \%)$ & $19(62 \%)$ & $37(66 \%)$ & \\
\hline 3 & $53(15 \%)$ & $4(13 \%)$ & $9(16 \%)$ & \\
\hline $\mathrm{T}$ & & & & 0.023 \\
\hline$<2 \mathrm{~cm}$ & $119(34 \%)$ & $7(22 \%)$ & $17(30 \%)$ & \\
\hline $2-5 \mathrm{~cm}$ & $189(54 \%)$ & $22(71 \%)$ & $29(52 \%)$ & \\
\hline$>5 \mathrm{~cm}$ & $43(12 \%)$ & $2(7 \%)$ & $10(18 \%)$ & \\
\hline HER-2 & & & & 0.7 \\
\hline Negative & $75(21 \%)$ & $3(18 \%)$ & $13(23 \%)$ & \\
\hline Positive & $98(28 \%)$ & $2(11 \%)$ & $15(27 \%)$ & \\
\hline Unknown & $178(51 \%)$ & $12(71 \%)$ & $28(50 \%)$ & \\
\hline Histology & & & & 0.001 \\
\hline DCIS & $68(19 \%)$ & $7(22 \%)$ & $12(22 \%)$ & \\
\hline IDC & $227(65 \%)$ & $23(74 \%)$ & $41(73 \%)$ & \\
\hline ILC & $56(16 \%)$ & $1(4 \%)$ & $3(5 \%)$ & \\
\hline
\end{tabular}

HER2, human epidermal growth factor receptor 2; IDC, Invasive ductal carcinoma; DCIS, ductal carcinoma in situ ; ILC, Invasive lobular carcinoma; $\mathrm{T}$, size of the original Tumour ue; N, lymph Nodes that are involved, M, Metastasis (spread of cancer from one part of the body to another).

those with synchronous BC, which indicates that survival was significantly higher $(p$ value $=0.022)$ in metachronous type compared to synchronous type (Log-rank Test chi2 $=3.54, p=0.038$; Figure 1 curve $(B))$.

5 -year survival rate in patients with $\mathrm{BC}$ significantly correlated with the type of breast tissue that the tumor had originated from. The highest survival rate was reported in patients whose lobule tissue of the breast was involved; on the other hand, the lowest survival rate has been reported among patients with involvement of interstitial
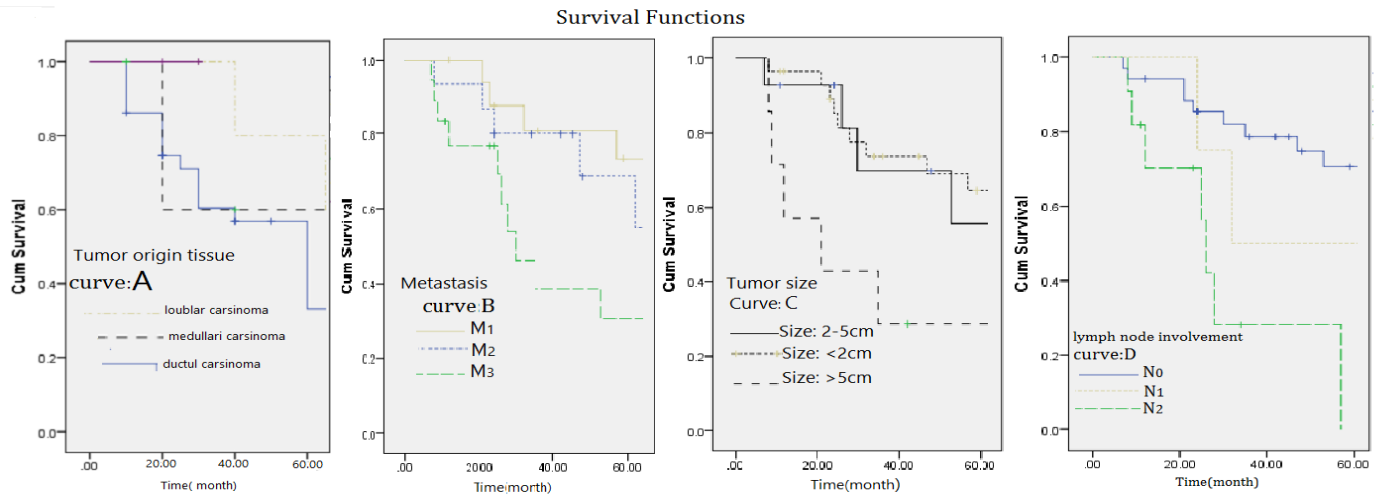

Figure 2: A) The curve comparing survival rate in patients with breast cancer based on type of breast tissue involved. B) The curve comparing survival rate in patients with breast cancer based on the stage of disease tissue involvement. C) The curve comparing survival rate in patients with breast cancer based on tumor size. D) The curve comparing survival rate in patients with breast cancer based on lymph node involvement rate. 
tissue (connective and adipose tissues). (Figure 2, curve A; Log-rank Test chi2 $=9.55, \mathrm{p}=0.0001$ ).

5 -year survival rate was reported to be significantly higher in patients that were diagnosed in the initial stage of tissue involvement (M1) compared to M2 and M3 stages (Figure 2, curve B; Log-rank Test chi2 $=11.54, \mathrm{p}=0.001$ ).

This study also showed a significant correlation between tumor size and survival of the patients after 5 years and as can be seen in curve $\mathrm{C}$ of Figure 2, the highest survival rate has been reported in $\mathrm{BC}$ patients with a tumor size less than $2 \mathrm{~cm}$ and the lowest survival rate was associated with sizes over $5 \mathrm{~cm}$. In this study, the correlation of 5-year survival rate in patients with $\mathrm{BC}$ with degree of lymph node involvement was also evaluated and the rate of 5-year survival was significantly higher in patients whose lymph node involvement rate was diagnosed to be in N1 stage compared to patients whose lymph node involvement was diagnosed to be in N2 and N3 stages. Survival curve of the patients based on lymph node involvement is shown in curve D of Figure 2 (Log-rank Test chi2 $=8.87, \mathrm{p}=0.027$ ).

\section{Discussion}

In individuals with $\mathrm{BC}$, there are factors that affect the survival rate of the patients after being diagnosed with cancer. Therefore, evaluating these factors has special importance for improving their quality of life and enhancing the effectiveness of treatment. The correlation of various factors with rate of survival after BC diagnosis has been evaluated and shown in different studies. For instance, Roder et al., (2012) showed that tumor size, stage of tumor, involvement of lymph nodes, estrogen receptor (ER) status, vascular status and being diffused correlate with survival rate after being diagnosed with BC. Dong et al., (2014) added menopausal status, and metastasis to armpit lymph nodes and the adjacent region to the factors correlating with survival after BC development (Dong et al., 2014). The results of Vaz-Luis study in 2012 showed that hormone receptors (HR) are among the factors correlating with survival rate after developing BC (Vaz-Luis et al. 2012). The pattern of hormone receptors is also one of the factors related to mortality following $\mathrm{BC}$ and in individuals with HR+/HER2+ and HR-/ HER 2+ patterns, the risk of death is significantly higher (Álvarez-Bañuelos et al., 2016). In our study, contrary to the study by Álvarez-Bañuelos et al. no significant correlation was reported between HER 2 receptors and rate of survival. There is controversy regarding the correlation of age with survival after being affected with $\mathrm{BC}$ and there are contradicting results. Some studies consider aging as a factor that leads to an increase in mortality after being affected with BC (Schonberg et al., 2010); yet, others dismiss this claim (Álvarez-Bañuelos et al., 2016). Although family history is among the definite risk factors of developing $\mathrm{BC}$, its correlation with survival rate after being affected with $\mathrm{BC}$ is not confirmed and is a matter of debate. Some studies assume presence or absence of a family history irrelevant (Malone et al., 2011) and other studies believe it is relevant (Chang et al., 2009) to survival rate after being affected with BC.
Auxiliary treatment such as chemotherapy (Goelet al., 2010 ; Hortobagyi, 2012) and hormone therapy (Group 2005; Sestak et al., 2013) are among factors improving survival rate after development of BC. In our study, in addition to evaluating the correlation of tumor size, staging, and metastasis to lymph nodes, the correlation of the $\mathrm{BC}$ being unilateral or bilateral with 5-year survival rate was evaluated for the first time, which can be considered as a significant point regarding this study. The results of our study showed that if the time interval between cancer development in affected and initially nonaffected breasts are more than 6 months (metachronous), 5 -year survival rate is significantly higher than the state in which the time interval between affected and initially non-affected breasts developing cancer being 6 months or less (synchronous). The probable explanation for this result can be that in the synchronous state, the individual bears 2 cancerous breasts for a longer time and therefore, the disease is more severe, there are more complications, and consequently, mortality rate will be higher. Our study showed that survival rate in patients with unilateral BC is significantly higher than the survival rate in patients with bilateral cancer. This difference in survival rate can be due to the higher rate of involvement and therefore, more need for treatment. The results of this study clearly showed that the number of survived patients whose cancer originated from lobule tissue (milk producing glands) has been significantly higher; while survival rate was reported to be much lower in patients whose tumor origin was the interstitial tissue (connective and adipose tissues) and the results of the study by Schnitt et al., (1986) also is consistent with our results.

The results of our study regarding evaluation of tumor size, staging, and metastasis to lymph nodes showed that patients whose tumor size was less than $2 \mathrm{~cm}$, the tumor was in a lower stage and had less metastasis to lymph nodes, had a higher rate of 5-year survival compared to other patients, which is consistent with the findings of a study by Álvarez-Bañuelos et al., (2016) performed. As this research team expressed, the increase in survival rate of these cases depends on early determination of a proper treatment strategy which increases effectiveness of treatment. It is obvious that delay between suspicion to cancer development and final diagnosis can affect clinical outcomes including mortality (Bright et al., 2011; Gagliato et al., 2014). Since the sooner the diagnosis is made, the lower the size, stage, and metastasis of tumor are, 5-year survival rate after diagnosis will be higher, which can be a logical explanation for the findings of our study.

\section{Authors' contributions}

All authors passed four criteria for authorship contribution based on recommendations of the International Committee of Medical Journal Editor.

\section{Conflict of interest}

There was no conflict of interest.

\section{References}

Álvarez-Bañuelos M, Rosado-Alcocer L, Morales-Romero J, 
Román Álvarez L, Guzmán-García R (2016). Prognostic factors associated with survival in women with breast cancer from Veracruz Mexico. J Cancer Sci Ther, 8, 92-8.

Balabram D, Turra CM, Gobbi H (2013). Survival of patients with operable breast cancer (Stages I-III) at a Brazilian public hospital-a closer look into cause-specific mortality. BMC Cancer, 13, 434.

Bright K, Barghash M, Donach M, et al (2011). The role of health system factors in delaying final diagnosis and treatment of breast cancer in Mexico City, Mexico. Breast J, 20, 54-9.

Brown ML, Lipscomb J, Snyder C (2001). The burden of illness of cancer: economic cost and quality of life. Ann Rev Public Health, 22, 91-113.

Chang ET, Milne RL, Phillips KA, et al (2009). Family history of breast cancer and all-cause mortality after breast cancer diagnosis in the breast cancer family registry. Breast Cancer Res Treat, 117, 167-76.

Dong G, Wang D, Liang X, et al (2014). Factors related to survival rates for breast cancer patients. Int J Clin Exp Med, 7, 3719-24.

Dorak MT, Karpuzoglu E (2012). Gender differences in cancer susceptibility: An inadequately addressed issue. Front Genet, 3, 268.

Gagliato DD, Gonzalez-Angulo MA, Lei X, et al (2014). Clinical impact of delaying initiation of adjuvant chemotherapy in patients with breast cancer. J Clin Oncol, 32, 735-44.

Goel MK, Khanna P, Kishore J (2010). Understanding survival analysis: Kaplan-Meier estimate. Int J Ayurveda Res, 1, 274.

Chia S, Bryce C, Gelmon K (2005). Effects of chemotherapy and hormonal therapy for early breast cancer on recurrence and 15-year survival: an overview of the randomised trials. Lancet, 365, 1687-1717.

Hortobagyi G, Minckwitz G, Untch M, et al (2012). Definition and impact of pathologic complete response on prognosis after neoadjuvant chemotherapy in various intrinsic breast cancer subtypes. Clin Oncol, 30, 1796-1804.

Jemal A, Bray F, Center MM, et al (2011). Global cancer statistics. CA Cancer J Clin, 61, 69-90.

Malone KE, Daling J, Doody DR, et al (2011). Family history of breast cancer in relation to tumor characteristics and mortality in a population-based study of young women with invasive breast cancer. Cancer Epidemiol Prev Bio (cebp), 781, 2011.

Nishimura R, Osako T, Nishiyama Y, et al (2013). Evaluation of factors related to late recurrence-later than 10 years after the initial treatment-in primary breast cancer. Oncology, 85, $100-10$

Roder DM, de Silva DP, Zorbas HM, et al (2012). Age effects on survival from early breast cancer in clinical settings in Australia. ANZ J Surg, 82, 524-8.

Schnitt SJ, Hayman J, Gelman R, et al (1996). A prospective study of conservative surgery alone in the treatment of selected patients with stage I breast cancer. Cancer, 77, 1094-100.

Schonberg MA, Marcantonio E, Li D, et al (2010). Breast cancer among the oldest old: tumor characteristics, treatment choices, and survival. J Clin Oncol, 28, 2038-45.

Sestak L, Dowsett M, Zabaglo L, et al (2013). Factors predicting late recurrence for estrogen receptor-positive breast cancer. J Natl Cancer Inst, 105, 1504-11.

Vakili M, Pirdehghan A, Adimi M, Sadeghian M, Akhondi M (2014). Epidemiology and trend of cancer in Yazd, a central province of Iran, 2005-2009. J Res Health Sci, 14, 210-13.

Vaz-Luis I, Ottesen RA, Hughes ME, et al (2012). Impact of hormone receptor status on patterns of recurrence and clinical outcomes among patients with human epidermal growth factor-2-positive breast cancer in the national
5-Year Survival Rates and Survival Predictors in Patients

comprehensive cancer network: a prospective cohort study. Breast Cancer Res, 14, R129.

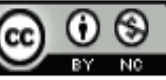

This work is licensed under a Creative Commons AttributionNon Commercial 4.0 International License. 